\title{
Wearable-band type Visible-Near Infrared (VIS-NIR) optical sensor for Non-invasive Blood Glucose Monitoring
}

\author{
Vega Pradana Rachim and Wan Young Chung \\ Department of Electronic Engineering, Pukyong National University, Busan 48513, South Korea, \\ wychung@pknu.ac.kr
}

\begin{abstract}
In this study, a combination of Visible and Near Infrared (Vis-NIR) optical-based wearable sensor was proposed as an alternative design for the non-invasive blood glucose monitoring system. A unique information of the pulsatile to continuous components of the arterial blood volume pulsation and the capillaries blood volume during the change of glucose concentration in tissue has been exposed using reflected light signal in multiple optical wavelengths collected from subject's wrist. Thus, an in-vitro experiment which enclosed six healthy subjects in a two-hour modified carbohydrate-rich meals tests has been conducted as an initial system-test. Results showed that the average correlation coefficient $(\mathrm{Rp})$ between predicted and reference glucose value from all the tested subjects reached 0.85 , with the standard error of prediction (SEP) of $7.70 \mathrm{mg} / \mathrm{dL}$. Moreover, the clinical significance analysis using Clarke error grid (CEG) was also handled, with the results of all data points fell within the clinically acceptable region (Region A: $100 \%$ ).
\end{abstract}

Key words: Non-invasive glucose monitoring, Biomedical Optics, Wearable Sensors, In-vitro, VisibleNear Infrared.

\section{Introduction}

Non-invasive blood glucose monitoring (BGM) play an important role in the control of diabetic patient's glucose level and healthy subjects. A daily non-invasive system can be useful for healthy subjects to control their quality of life and to prevent the diseases, as there is no cure for diabetes. However, an existing system which relies on invasive and minimally-invasive technique are painful, discontinues, and have an expensive-limited sensor lifespan. Moreover, some other non-invasive method such as sweat-based sensor patch usually utilized an expensive material and has a measurement delay time around $15 \mathrm{~min}$ to collect the sweat. Therefore in this study, a fully wearable, costefficient and compact optical sensor was proposed as a continuous non-invasive BGM system.

\section{System Design}

Fig.1 shows the block diagram of our proposed system, including the example of the received signal in multiple optical wavelengths. The proposed sensor module contains two visible (Vis) and two near-infrared (NIR) LED drivers to emit Vis-NIR light in four optical wavelengths of $535 \mathrm{~nm}, 660 \mathrm{~nm}, 850 \mathrm{~nm}$, and $950 \mathrm{~nm}$, respectively. Furthermore, a photodiode (PD) and trans-impedance amplifier (TIA) used to detect and convert the reflected light into a voltage from the subject's skin. The collected raw signal from each LED is converted to the digital data by the analog to digital converter (ADC). In this system, MCU has been used for ADC, and also for PD sampling and LED timing control. Thus, each LED turns on alternately with $t_{\text {on }}=250 \mu \mathrm{s}$ and collected by the PD in $t_{s}=2 \mathrm{~ms}$.

As mentioned before, in this study a combined Vis-NIR optical-wavelength band that lies between 500 and $1000 \mathrm{~nm}$ has been used. The method is based on the assumption that glucose concentration in tissue affects cutaneous microcirculation and light scattering [1], thus the wavelength selection did not belong to any glucose absorption band. Moreover, the blood glucose concentration prediction model are completed using multiple predictors from the derived optical density $(\triangle \mathrm{OD})$ and a Kaiser-Teager Energy (KTE) of a direct-arterial pulsatile blood volume changes (NIR-light photoplethysmography (PPG) signals) and indirect-capillaries density changes (Vis-light PPG signals). 
Tab. 1: System performance comparison when using different input or predictor.

\begin{tabular}{|c|c|c|c|c|}
\hline Input / Predictor & SEP (mg/dL) & $R_{p}$ & CEG A (\%) & CEG B (\%) \\
\hline Visible (535 \& 660 nm) & 12.42 & 0.46 & 91.7 & 8.3 \\
\hline NIR (850 \& 950 nm) & 10.51 & 0.63 & 95.83 & 4.17 \\
\hline $\begin{array}{c}\text { Vis-NIR (535,660,850, } \\
\text { \& 950 nm) }\end{array}$ & 7.70 & 0.85 & 100 & 0 \\
\hline
\end{tabular}

\section{Experimental Results}

To verify the performance of our proposed system, 12 test was conducted on six healthy subjects (range 22-30, two women and four men), collecting a total of 72 finger-prick blood glucose concentration data measured by HealthProTM (OSANG Healthcare, Korea) ranging from 89 to $152 \mathrm{mg} / \mathrm{dL}$. Each invasiveblood sample test is measured every 20 minutes for a total $120 \mathrm{~min}$ of the measurement time. An optical data were collected from each subject left wrist for $60 \mathrm{~s}$ directly after the invasive-blood sample test. During this experiment, PPG signals are recorded in four optical-wavelength. Thus an $A C$ component $\left(\Delta I_{\lambda}\right)$, and the DC component of the PPG signal $\left(I_{\lambda}\left(t_{i}\right)\right)$ are extracted, and $\triangle O D_{\lambda}$ that correspond to the difference of the optical density in the specific wavelength is calculated as eq.1 [2]:

$$
\Delta O D_{\lambda}=\log \frac{I_{\lambda}\left(t_{i+1}\right)}{I_{\lambda}\left(t_{i}\right)}=\log \left(1+\frac{\Delta I_{\lambda}\left(t_{i}\right)}{I_{\lambda}\left(t_{i}\right)}\right)
$$

The overall comparison performance is shown in Table 1. In our experiment, multiple input/predictor were compared. Therefore, as expected, a model which created from Vis-NIR data is overcome the other model which only using either Vis input data or NIR input data. Moreover, we randomly selected $70 \%$ of data to create a PLS-based calibration model, while the $30 \%$ of data used for testing/prediction. Thus the SEP between the predicted glucose $(G p)$ and the reference glucose $(G r f)$ are measured in the calibration model by the following equation:

$$
=\sqrt{\frac{\sum_{i=1}^{n}\left\{\left(G p_{i}-\overline{G p}\right)-\left(G r f_{i}-\overline{G r f}\right)\right\}^{2}}{n}}
$$

Therefore, our system achieved the $R_{p}>0.85$ and SEP $<7.70 \mathrm{mg} / \mathrm{dL}$. CEG analysis also conducted on our prediction model, and we found that all data points fell within the clinically acceptable region (Region A: $100 \%$ ) as seen in Fig.2. Finally, a difference between predicted and measured blood glucose concentration is analyzed using Bland-Altman plot, see Fig.3. Thus we found a bias and precision of $1.00 \pm$ 7.75 (mean \pm SD).

\section{References}

[1] S. J. Yeh, C. F. Hanna, O. S. Khalil, Clinical Chemistry. 2003 Jun 1;49(6):924-34; doi: 10.1373/49.6.924.
[2] K. I. Yamakoshi, Y. Yamakoshi, Journal of Biomedical Optics. 2006 Sep;11(5):054028; doi: 10.1117/1.2360919.
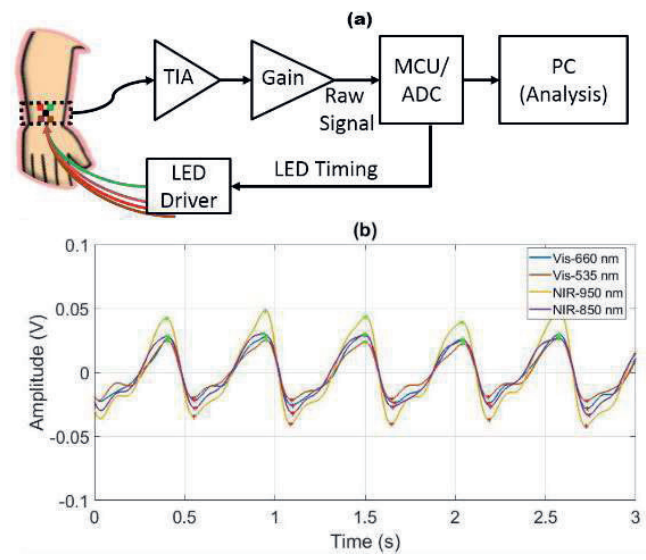

Fig. 1(a) Block diagram of the proposed system with (b) the example of the received signal in four different optical wavelength.

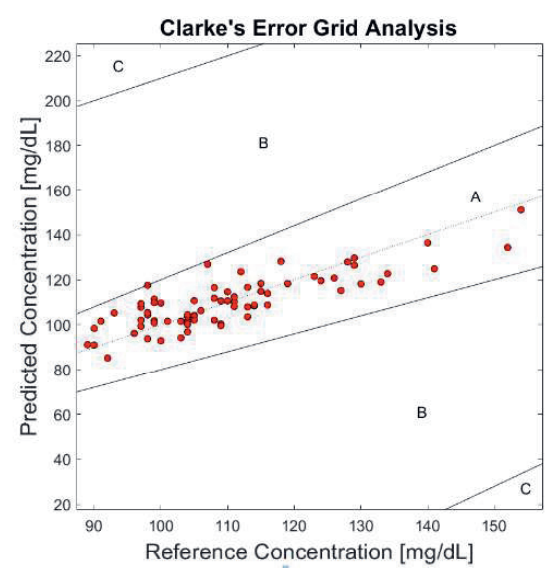

Fig. 2. Clarke Error Grid analysis of the proposed system.

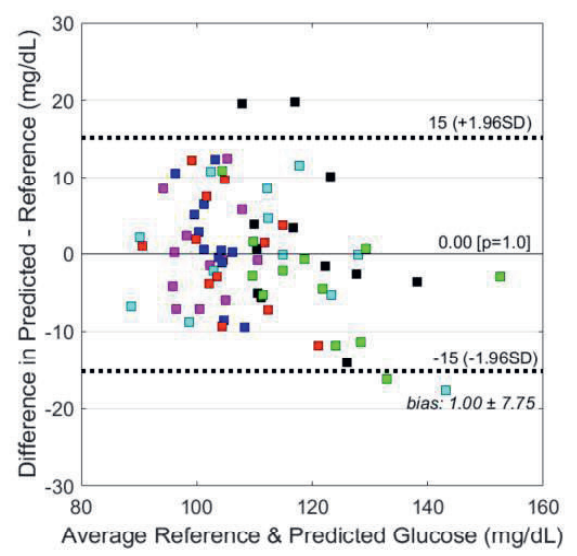

Fig. 3. Bland-Altman plot for system performance analysis 